\title{
MS30-01 | Solid-State ChiRal Resolution via Metal Complexation
}

Grepioni, Fabrizia (University of Bologna, Bologna, ITA)

Spontaneous chiral resolution upon ionic co-crystals (ICCS) formation is observed reacting the amino acid histidine or proline with lithium halides. The $\mathrm{Li}^{+}$cations selectively link to molecules of the same chirality, forming enantiopure chains, resulting in a chiral resolution process in the solid-state via conglomerate formation.

One possible reason for chiral preference in lithium ICCS could be the tetrahedral geometry around the lithium cations, which favours the coordination of molecules of the same handedness. Complexation of enantiopure $S$ etiracetam (levetiracetam) and of racemic RS-etiracetam to zinc in the form of their $\mathrm{ZnCl}_{2}$ salts has also been investigated, as zinc is known to favour tetrahedral coordination. By varying the stoichiometric ratio it is possible to "switch" reversibly from a stable racemic compound to a conglomerate. Co-crystallization with metal ions favouring tetrahedral coordination can thus be successfully used to obtain chiral selectivity and conglomerate formation from racemic compounds.

[1] D. Braga, L. D. Esposti, K. Rubini, O. Shemchuk and F. Grepioni, Cryst. Growth Des., 2016, 16, 7263-7270.

[2] O. Shemchuk, L. Degli Esposti, F. Grepioni and D. Braga, CrystEngComm, 2017, 19, 6267-6273

[3] O. Shemchuk, L. Song, K. Robeyns, D. Braga, F. Grepioni and T. Leyssens, Chem. Commun., 2018, 54, $10890-$ 10892.

[4] L. Song, O. Shemchuk, K. Robeyns, D. Braga, F. Grepioni, and T. Leyssens, Cryst.Growth Des., 2019, DOI:10.1021/acs.cgd.9b00136 